\title{
CANCER
}

\section{Galectin-8 expression decreases in cancer compared with normal and dysplastic human colon tissue and acts significantly on human colon cancer cell migration as a suppressor}

\author{
N Nagy, Y Bronckart, I Camby, H Legendre, H Lahm, H Kaltner, Y Hadari, P Van Ham, \\ P Yeaton, J-C Pector, Y Zick, I Salmon, A Danguy, R Kiss, H-J Gabius
}

See end of article for authors' affiliations

Correspondence to: R Kiss, Laboratory of Histopathology, Faculty of Medicine, Université Libre de Bruxelles, 808 route de Lennik, 1070 Brussels,

Belgium; rkiss@ulb.ac.be

Accepted for publication 18 May 2001

\begin{abstract}
Background and aims: Galectins are $\beta$-galactoside binding proteins. This ability may have a bearing on cell adhesion and migration/proliferation in human colon cancer cells. In addition to galectins- 1 and -3 studied to date, other members of this family not investigated in detail may contribute to modulation of tumour cell features. This evident gap has prompted us to extend galectin analysis beyond the two prototypes. The present study deals with the quantitative determination of immunohistochemical expression of galectin-8 in normal, benign, and malignant human colon tissue samples and in four human colon cancer models (HCT-15, LoVo, CoLo201, and DLD-1) maintained both in vitro as permanent cell lines and in vivo as nude mice xenografts. The role of galectin-8 (and its neutralising antibody) in cell migration was investigated in HCT-15, LoVo, CoLo201, and DLD-1 cell lines.

Methods: Immunohistochemical expression of galectin-8 and its overall ability to bind to sugar ligands (revealed glycohistochemically by means of biotinylated histochemically inert carrier bovine serum albumin with $\alpha$ - and $\beta$-D-galactose, $\alpha$-D-glucose, and lactose derivatives as ligands) were quantitatively determined using computer assisted microscopy. The presence of galectin- 8 mRNA in the four human colon cancer cell lines was examined by reverse transcriptase-polymerase chain reaction. In vitro, cellular localisation of exogenously added galectin-8 in the culture media of these colon cancer cells was visualised by fluorescence microscopy. In vitro galectin-8 mediated effects (and the influence of its neutralising antibody) on migration levels of living HCT-15, LoVo, CoLo201, and DLD-1 cells were quantitatively determined by computer assisted phase contrast microscopy.

Results: A marked decrease in immunohistochemical expression of galectin-8 occurred with malignancy development in human colon tissue. Malignant colon tissue exhibited a significantly lower galectin-8 level than normal or benign tissue colon cancers; those with extensive invasion capacities (T3-4/N+/M+) harboured significantly less galectin-8 than colon cancers with localised invasion capacities (T1-2/N0/MO). The four experimental models (HCT-15, LoVo, CoLo201, and DLD-1) had more intense galectin- 8 dependent staining in vitro than in vivo. Grafting the four experimental human colon cancer models onto nude mice enabled us to show that the immunohistochemical expression of galectin-8 was inversely related to tumour growth rate. In vitro, galectin- 8 reduced the migration rate of only those human experimental models (HCT-15 and CoLo201) that exhibited the lowest growth rate in vivo.

Conclusions: Expression of galectin-8 correlated with malignancy development, with suppressor activity, as shown by analysis of clinical samples and xenografts. In vitro, only the two models with low growth rates were sensitive to the inhibitory potential of this galectin. Future investigations in this field should involve fingerprinting of these newly detected galectins, transcending the common focus on galectins- 1 and -3 .
\end{abstract}

$\mathrm{P}$ lant lectins and monoclonal antibodies have been instrumental in detecting alterations in the profiles of glycans in cell glycoconjugates on malignant transformation. Initially of phenomenological value, detection of glycan-epitope specific proteins (lectins) in tumour cells indicated the presence of a productive protein-carbohydrate recognition system. ${ }^{12}$ With the ensuing elaboration of the structural and functional aspects of mammalian lectins it has become possible to thoroughly explore correlations between lectin expression and tumour features based on the hypothesis of glycan functionality. ${ }^{3-5}$ Owing to their spatial accessibility, $\beta$-galactosides at the termini of $\mathrm{N}$ - and O-glycan antennae offer suitable docking sites for endogenous lectins. Indeed, one family of lectins - the galectins ${ }^{6-13}$ - is devoted to interacting with such sites. To date, 12 members of the galectin family have been classified based on the presence of invariant sequence motifs. These proteins share a common topology of the carbohydrate recognition domain (CRD) with high sequence homology scores in intermammalian comparisons, secretion via a non-classical pathway, and interaction with the extracellular glycans laminin and fibronectin, $\alpha 1 \beta 1$ and $\alpha 7 \beta 1$ integrins, and other cell surface components such as CD2, CD3, CD4, CD7, CD43, CD45, gastrointestinal mucins, and distinct neutral glycolipids or ganglioside GMl (for review,

Abbreviations: $A S$, average speed; $C C D$, coupled charge device; $C R D$, carbohydrate recognition domain; LI, labelling index; MOD, mean optical density; MRDO, maximum relative distance to the origin; RT-PCR, reverse transcriptase-polymerase chain reaction. 

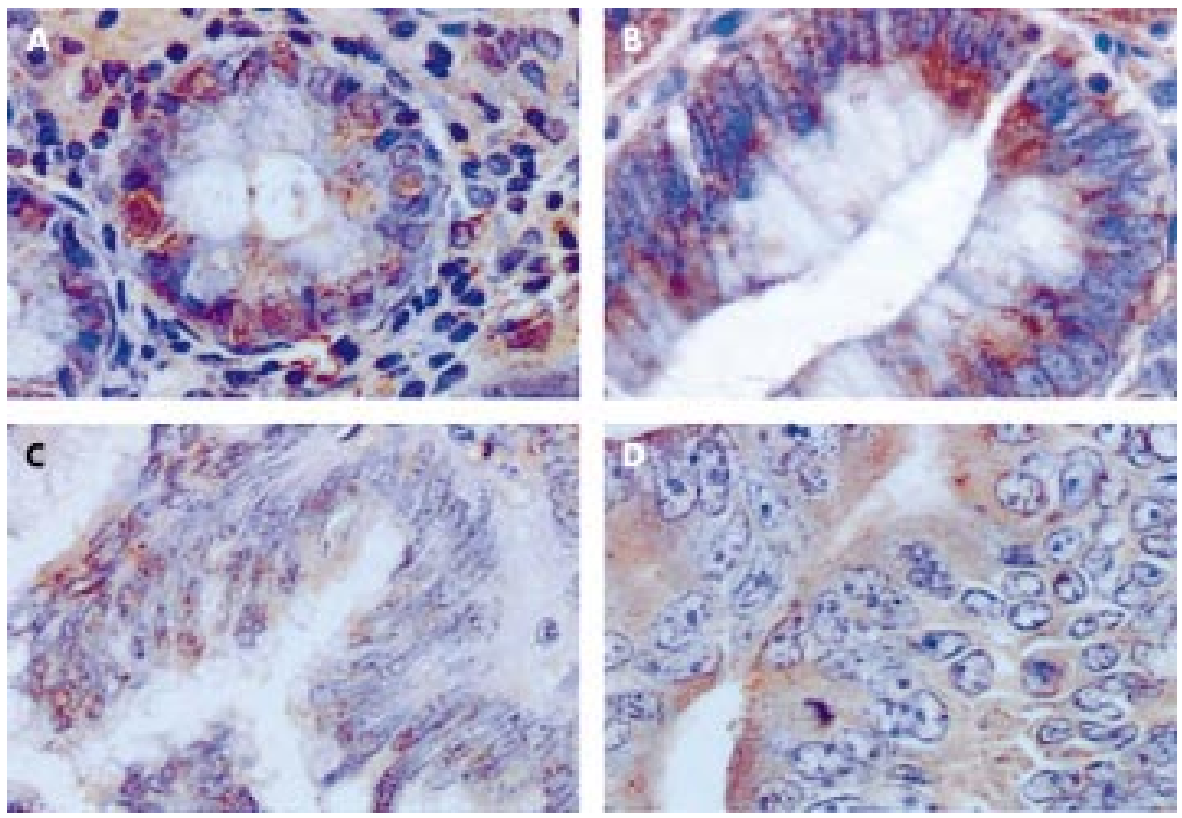

Figure 1 Morphological illustration $(G \times 400)$ of immunohistochemical expression of galectin-8 in glandular tissue from a normal colon (A), adenoma (B), locally invasive carcinoma (C), and an extensively invasive carcinoma (D).

$\left.\operatorname{see}^{6-13}\right)$. In addition to mediation of cell contacts, galectin binding has been shown to affect cell growth with cell type dependent modulation of proliferation and induction of apoptosis in studies using an exogenous supply of galectin and genetically defective mice. Cyto- and histochemical investigations have also directed attention to the nuclear and cytoplasmic localisation of galectin- 1 and -3 in various cell types, such as fibroblasts or chondrocytes in vitro and in vivo. ${ }^{6-13}$ With respect to colon tumour biology, the presence of galectins-1 and -3 has so far been investigated histopathologically using different methods and with conflicting results. ${ }^{8}{ }^{84}$ Two recent reports underscore the potential relevance of galectin-3 for colon cancer progression and metastasis. ${ }^{15}{ }^{16}$ Once there is an awareness of the functional redundancy and antagonism in the galectin family-for example pro- and antiapoptotic activities of galectins- $1 /-9$ versus galectin-3-to monitor cancer specimens it becomes crucial to extend beyond the two galectins analysed so far to obtain valid conclusions, an approach previously referred to as galectin fingerprinting. ${ }^{17} 18$ To this end, we carried out this study on colon cancer in vitro and in vivo using a new member of the galectin family.

We recently cloned a new mammalian galectin (designated galectin-8) from a rat liver cDNA expression library. ${ }^{19}$ It belongs to the subclass of tandem repeat type galectins with two CRDs connected by a 32 amino acid linker with structural homology to galectin-4. At the nucleic acid level, galectin- 8 is $50 \%$ homologous to galectin- 4 while at the amino acid level, these two galectins share $34 \%$ identity. ${ }^{19}{ }^{20}$ However, several important features distinguish galectin-8 from galectin-4: (i) unlike galectin- 4 , but similar to galectins- 1 and -2 , galectin- 8 contains four Cys residues; (ii) the link peptide of galectin- 8 is unique, with no similarity to galectin- 4 or -6 or the metalloproteinase sensitive tandem repeats of galectin-3; and (iii) the $\mathrm{N}$-terminal carbohydrate binding region of galectin- 8 contains an unusual W-G-E-I motif instead of the consensus W-G-E-R/K motif involved in sugar binding in all other galectins. ${ }^{19}{ }^{20}$ The similarity also extends to galectin-6, another member of the tandem repeat type subgroup with marked expression in the gastrointestinal tract, which has $83 \%$ amino acid identity with galectins -4 and $-6 .{ }^{19-22}$ Whereas galectin- 4 is expressed in about equal levels in the colon and small intestine, but much less so in the stomach, ${ }^{20-23}$ galectin-6 is expressed in approximately equal amounts throughout the mouse gastrointestinal tract. ${ }^{22}$ Northern blot analysis revealed that galectin- 8 is expressed in rat gastrointestinal tract tissue and also in the kidneys, cardiac muscle, lungs, and the brain. ${ }^{19}$

Interestingly, expression of insoluble galectin-4, also described as an adherent junction protein, has previously been shown to decrease during the development of malignancy in colon tissue from 18 patients. ${ }^{20}$ In contrast with galectin-4, galectin- 8 is soluble and secreted, in common with galectins- 1 and -3 , without the requirement for a signal peptide. To date, no studies on the cellular functions of this galectin in colon cancer have been carried out.

The aim of the present study was to investigate whether galectin- 8 plays a significant role in the biology of human colon cancers. Firstly, we quantitatively determined immunohistochemical expression of galectin- 8 in normal, dysplastic, and cancerous human colon tissue obtained after surgical resections or endoscopy. We then used four in vitro human colon cancer cell lines (HCT-15, LoVo, DLD-1, and CoLo201) in which we assessed the presence of galectin- 8 mRNA by reverse transcriptase-polymerase chain reaction (RT-PCR). Immunocytochemical and histochemical galectin-8 expression in the four in vitro colon cancer cells and four cell lines xenografted in vivo into nude mice was determined by computer assisted microscopy. As a role for galectin-4 in cell adhesion and/or cell motility has been intimated by cytochemical data in the human T84 colon cancer model, which was subsequently questioned because of its position exclusively on the apical surface of enterocytes, ${ }^{24}{ }^{25}$ we also characterised the influence of galectin- 8 on the motility (migration) level of the HCT-15, LoVo, DLD-1, and CoLo201 in vitro models using a computer assisted phase contrast microscope approach, as previously described..$^{26}$

\section{MATERIALS AND METHODS}

\section{Histopathological grading and clinical staging}

The clinical series of 41 human colon tissue specimens consisted of five controls, 10 adenomas, and 26 carcinomas. The normal control samples were from routine biopsies of colorectal areas without any signs of clinical abnormalities and were carried out in patients undergoing colonoscopy for examination or screening purposes. The 10 adenomas 


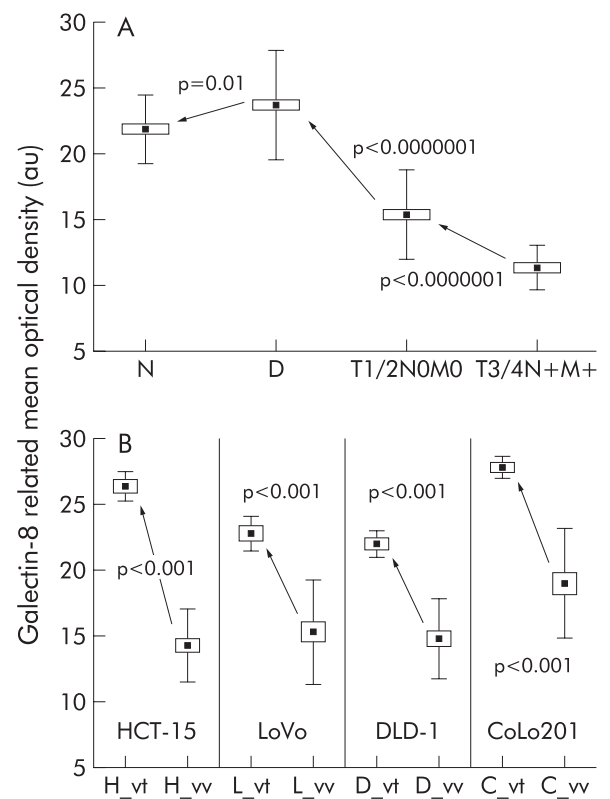

Figure 2 The 41 cases under study were grouped according to different histopathological and clinical variables, and galectin-8 concentration in epithelial tissue (measured as mean optical density) was thus quantitatively determined. (A) Data obtained for the norma $(N)$, dysplastic (D), locally invasive (T1 or T2 and NO and MO variables of the TNM staging system), and extensively invasive cancer (T3 or T4 and $\mathrm{N}+$ and $\mathrm{M}+$ ) cases. (B) Quantitative determination of the extent of galectin-8 expression in epithelial tissue analysed in four human colon cancer cell lines cultured in vitro or grafted subcutaneously into nude mice. Five distinct in vitro cell populations (obtained from five distinct in vitro passages) and five distinct in vivo xenografts were analysed for each of the four models (HCT-15, LoVo, DLD-1, and CoLo201). Ten microscope fields (G×200) were analysed for each tumour. All fields analysed for a given colon model were grouped together. For each of the four models under study, the " $x$ " $v$ vt symbol on the $x$ axis represents the in vitro cell line and the " $x$ "_ $v v$ is its corresponding in vivo xenograft. All data are presented as means (black squares), SEM (open rectangles), and SDs (bars).

included five cases with low dysplasia and five with severe dysplasia. Dysplasia grading was carried out according to a procedure previously described. ${ }^{28}$

Cancer staging was performed according to the conventional cancer staging system. ${ }^{29}$ The 26 cancers included four $\mathrm{T} 1$, five $\mathrm{T} 2$, nine $\mathrm{T} 3$, and eight $\mathrm{T} 4$ cases. Of these 26 cancers, 14 exhibited node invasions, and of these 14 node positive colorectal cancers, eight had additional liver metastases.

All specimens were fixed in buffered formalin before being further processed for paraffin embedding.

\section{Cell lines, culture media, and nude mice grafting procedure}

The four human colon cancer cell lines were obtained from the American Type Culture Collection (ATCC, Manassas, Virginia, USA). These four lines included the HCT-15 (ATCC code CCL225), LoVo (ATCC code CCL229), CoLo201 (ATCC code CCL224), and DLD-1 (ATCC code CCL221) models. We have previously detailed the cell kinetics of these four cell lines in addition to their growth sensitivity towards various hormones and/or growth factors ${ }^{30} 31$ and five plant lectins. ${ }^{32}$

Cells were cultured at $37^{\circ} \mathrm{C}$ in sealed (airtight) Falcon plastic dishes (Nunc, Gibco, Belgium) containing Eagle's minimal essential medium (Gibco) supplemented with $10 \%$ fetal calf serum. All media were supplemented with a mixture of 0.6 $\mathrm{mg} / \mathrm{ml}$ L-glutamine (Gibco), $200 \mathrm{IU} / \mathrm{ml}$ penicillin (Gibco), 200 $\mathrm{IU} / \mathrm{ml}$ streptomycin (Gibco), and $0.1 \mathrm{mg} / \mathrm{ml}$ gentamycin (Gibco). Fetal calf serum was heat inactivated for one hour at $56^{\circ} \mathrm{C}$.

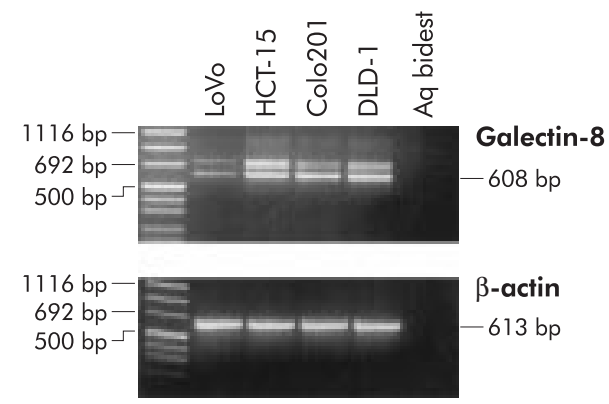

Figure 3 Galectin-8 gene expression by human colorectal carcinoma cell lines (HCT-15, LoVo, DLD-1, and Colo201). Reverse transcriptase-polymerase chain reaction analysis revealed the presence of two galectin-8 specific bands in all four cell lines.

In vivo nude mice xenografts were obtained from the HCT-15, LoVo, CoLo201, and DLD-1 in vitro cell lines using a procedure described previously with respect to grafting of human colon cancers into this type of nude mouse. ${ }^{33}$ Briefly, one million cells from each of the four cell lines were subcutaneously grafted into eight week old female nu/nu mice (21-23 g; Iffa Credo, IFFA Credo, Arbresle, France). Five nude mice were grafted for each of the four cell lines. At the 28th day post graft, the mice were sacrificed and the tumours removed. The size of each tumour was determined using calipers, thus enabling the two largest perpendicular diameters of each tumour to be measured. Tumour size was thus expressed as an "area" $\left.\left(\mathrm{mm}^{2}\right)\right)^{34}$ Each tumour was also weighed and we obtained a level of statistical significance of $\mathrm{p}<0.000001$ (Kendall Tau $=0.88$ ) between tumour size expressed as an area and tumour weight (data not shown). Once the measurements and weighings had been carried out, each tumour was fixed in buffered formalin $(4 \%)$ for four days, dehydrated, and embedded in paraffin wax.

Histochemical procedures for revealing the presence of galectin-8, and binding sites for carrier immobilised $\alpha$-galactose, $\beta$-galactose, $\alpha$-glucose, and lactose residues

Even when working with cell lines, we applied histochemical procedures throughout the study to avoid problems of membrane permeabilisation that may occur with cytochemical procedures. Thus with respect to the four cell lines cultured in vitro, we made cell pellets by centrifuging 10 million cells from each of the four cell lines for 10 minutes at $800 \mathrm{~g}$. These pellets were then fixed in buffered formalin (4\%) for four days, dehydrated, and embedded in paraffin wax. Three pellets were available for each of the four cell lines. All histochemical procedures were carried out as detailed previously. ${ }^{283}$ Briefly, $5 \mu \mathrm{m}$ thick sections were taken from each cell line pellet, xenograft, and clinical sample. Incubation with the various antibodies or synthetic probes was carried out at $25 \pm 1^{\circ} \mathrm{C}$ for 60 minutes with minimal background staining and at the dilutions described below for each antibody. The extent of the specifically bound antibodies or probes was visualised by avidin-biotin-peroxidase complex (ABC) kit reagents (Vector Labs, Burlingame, California, USA), with diaminobenzidine/ $\mathrm{H}_{2} \mathrm{O}_{2}$ as the chromogenic substrate. The antigalectin-8 polyclonal antibody, which was raised in a rabbit against the recombinant rat protein ( $80 \%$ homology to human protein), was produced and purified as described previously ${ }^{19}$ and used at a dilution of 1:500. Control tissue sections were incubated with the corresponding preimmune sera, with the second antibody only, or with the polyclonal antigalectin-8 antibody preabsorbed with recombinant galectin-8. The specificity of each antibody was checked by western blotting using galectins- $1,-3,-4$, and -7 as probes to detect any cross reactivity (data not shown). The control reactions for the biotinylated neoglycoproteins included competitive inhibition to ascertain 

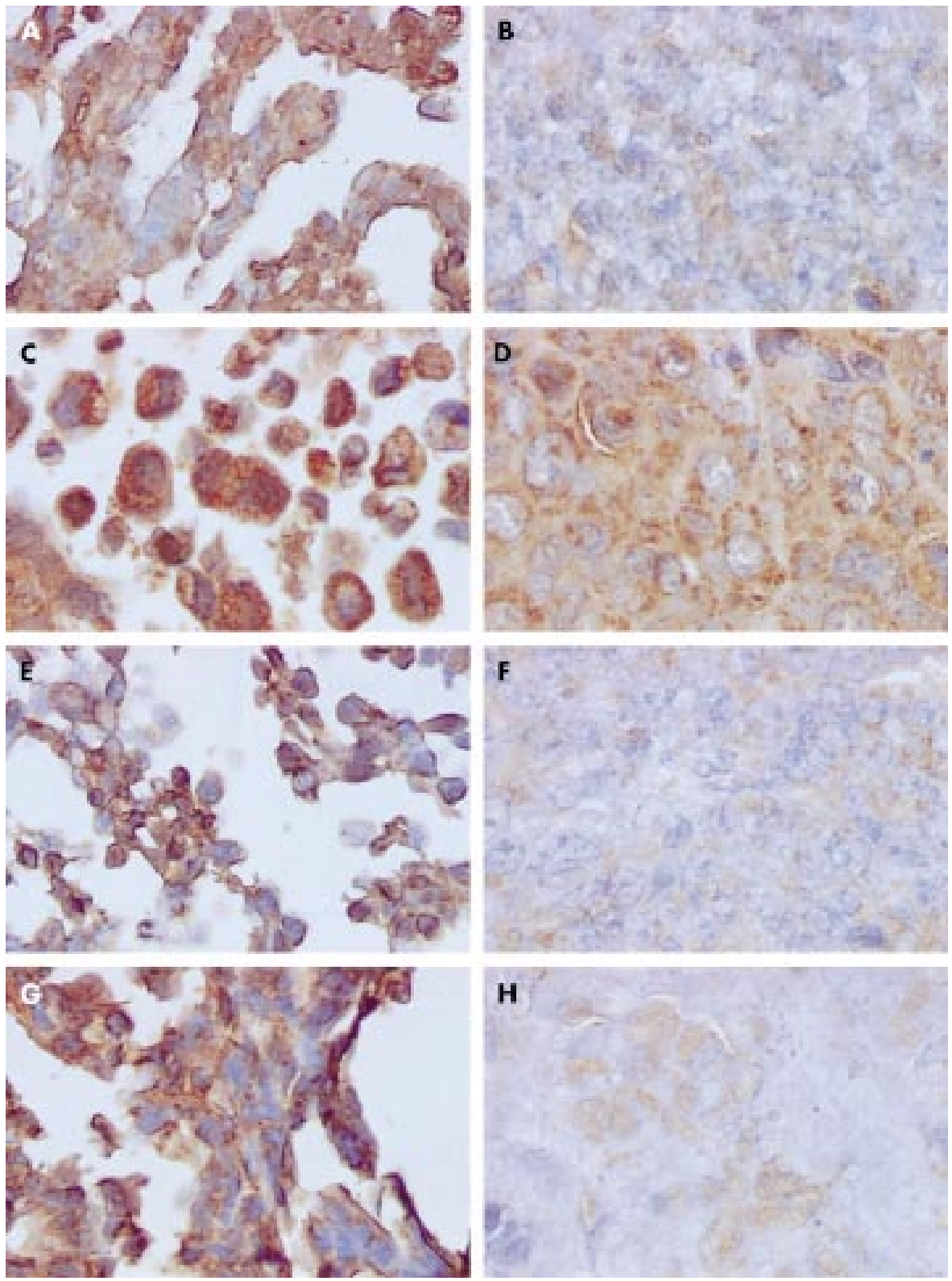

Figure 4 Morphological illustration $(\mathrm{G} \times 400)$ of immunohistochemically detected galectin-8 expression in the four human colon cancer cell lines cultured in vitro and in their corresponding in vivo xenografts. (A) In vitro HCT-15 cell line; (B) in vivo HCT-15 xenograft; (C) in vitro Colo201 cell line; $(D)$ in vivo CoLo201 xenograft; $(E)$ in vitro LoVo cell line; $(F)$ in vivo LoVo xenograft; $(G)$ in vitro DLD-1 cell line; and (H) in vivo DLD-1 xenograft.

sugar specificity, and omission of the incubation step with a labelled marker served to exclude any staining by the binding of kit reagents such as the mannose rich glycoproteins horseradish peroxidase and avidin. ${ }^{34}$ The neoglycoconjugates were synthesised and biotinylated, as described previously. ${ }^{35}$ Counterstaining was carried out with haematoxylin.

\section{Computer assisted microscopy for quantitative histochemistry}

Two variables were computed for each marker (a biotinylated neoglycoprotein or an antibody) using a SAMBA 2005 computer assisted microscope system (UNILOG, Grenoble, France) with a $20 \times$ (aperture 0.50) magnification lens. The labelling index (LI) refers to the percentage of tissue area specifically stained by a histochemical probe. Mean optical density (MOD) denotes staining intensity. The computer assisted system used to quantify histochemical staining is detailed elsewhere. ${ }^{36}$ The computer assisted microscope and related quantitative analyses were standardised as follows. A negative histological control slide (which was not exposed to either the biotinylated neoglycoprotein or the primary antibody) was analysed for each of the probes mentioned above, and for each type of biological sample under study - that is, the cell line pellets, xenografts, and clinical samples. The software used in the computer assisted microscope automatically subtracted the LI and MOD values of the negative control sample from each corresponding positive value. Special software was used to assist the microscope in checking any inherent shading in the coupled charge device (CCD) camera based systems, the glare phenomenon, and the level of precision of the linearity. Shading and glare were checked each week. Monitoring carried out by our computer assisted microscope showed that shading, glare, or linearity did not significantly alter our results (data not shown).

Ten areas of between 60000 and $120000 \mathrm{\mu m}^{2}$ were scanned for each of the biological specimens (that is, the 41 clinical 

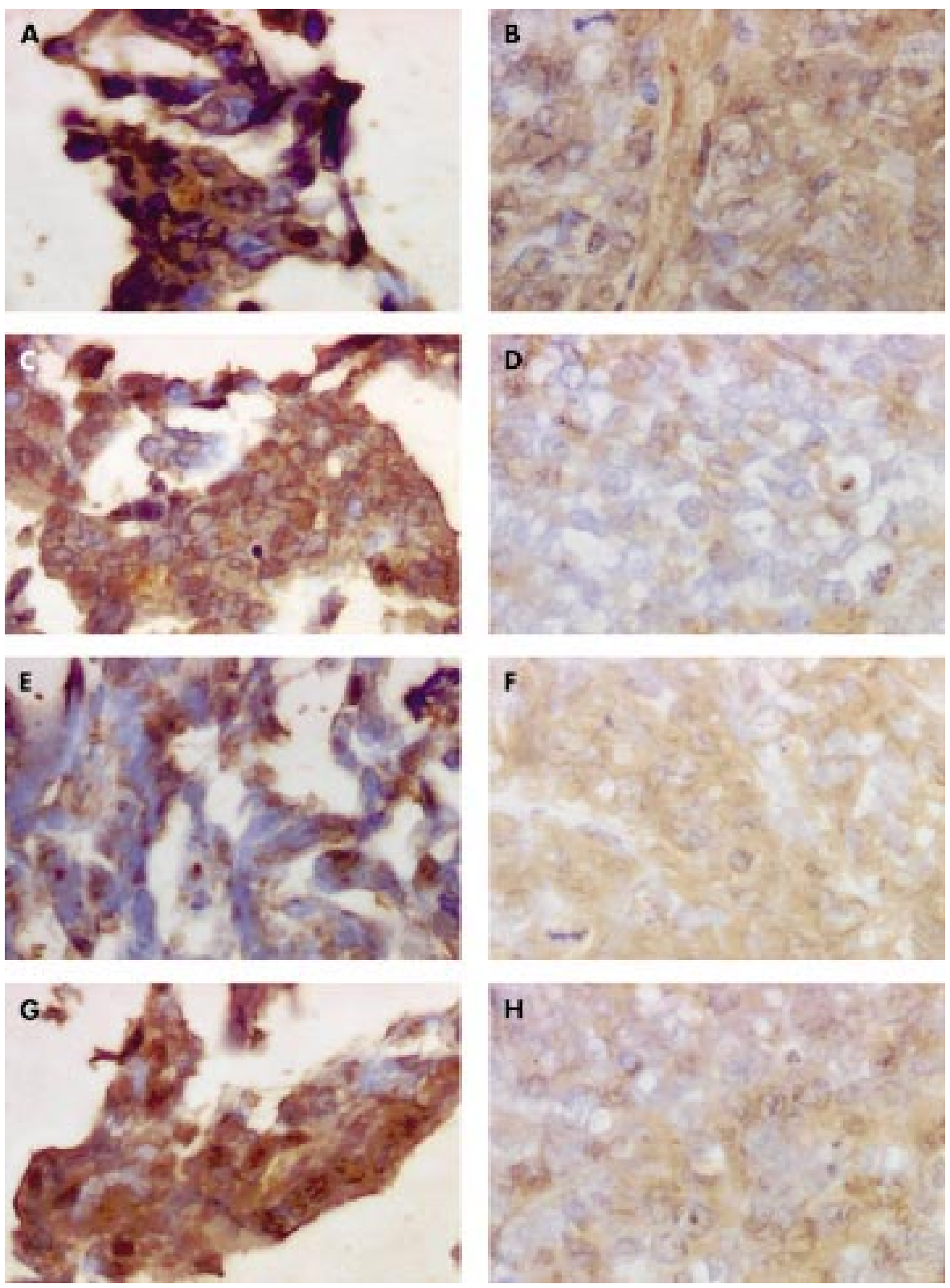

Figure 5 Morphological illustration $(G \times 400)$ of glycohistochemically detected binding sites for four distinct oligosaccharide moieties in the HCT-15 human colon cancer model. (A) (in vitro specimen) and (B) (in vivo specimen) relate to the $\alpha$-Gal-neoglycoconjugate, (C) (in vitro specimen) and (D) (in vivo specimen) to the $\beta$-Gal-neoglycoconjugate, (E) (in vitro specimen) and (F) (in vivo specimen) to the $\beta$-Glu-neoglycoconjugate, and $(G)$ (in vitro specimen) and $(H)$ (in vivo specimen) to the Lac-neoglycoconjugate.

samples, triplicate in vitro pellets, and the five in vivo xenografts available for each cell line) under study with each marker investigated here. Only epithelial tissue was analysed, and a computer mouse linked to the software on the computer assisted microscope enabled all non-epithelial tissue to be removed from the video control before digitising the histological field to be analysed.

\section{RT-PCR technique for galectin-8 mRNA detection in the four human colon cancer cell lines}

Cells were grown to subconfluence in Petri dishes and lysed by addition of Tris-Pure isolation reagent (Roche Diagnostics, Mannheim, Germany). Total RNA was prepared according to the manufacturer's recommendation, and $2.5 \mu \mathrm{g}$ of RNA were used as a template for cDNA synthesis. Prior to first strand synthesis, RNA was incubated with DNAse I ( $10 \mathrm{U} / \mathrm{\mu l}$; Roche Diagnostics, Mannheim, Germany) for 30 minutes at $37{ }^{\circ} \mathrm{C}$. The enzyme was heat inactivated at $75^{\circ} \mathrm{C}$ for 10 minutes. Reverse transcription was performed for 60 minutes at $37^{\circ} \mathrm{C}$ in RT buffer (50 mM Tris/ $\mathrm{HCl}, \mathrm{pH} 8.3,75 \mathrm{mM} \mathrm{KCl}, 3 \mathrm{mM} \mathrm{MgCl}_{2}$ ), $10 \mathrm{mM}$ DTT, dNTPs ( $1 \mathrm{mM}$ each), random hexamer primers (30 ng/pl), and $20 \mathrm{U}$ M-MLV reverse transcriptase (Gibco, Karlsruhe, Germany). The reaction was terminated by incubation for 10 minutes at $95^{\circ} \mathrm{C}$. The integrity of cDNA was confirmed by $\beta$-actin specific PCR analysis, as previously described. ${ }^{37}$

The following primers were used for the RT-PCR analysis: galectin-8 sense $5^{\prime}$ GTT GTC CTT AAA CAA CCT ACA G $3^{\prime}$ and galectin-8 antisense $5^{\prime}$ TAA CGA CGA CAG TTC GTC CAG 3'. All PCR analyses were carried out in a $20 \mu \mathrm{l}$ reaction medium containing $2 \mu \mathrm{l}$ cDNA, $0.5 \mathrm{U}$ Taq polymerase (MWG, Munich, Germany), $50 \mathrm{mM} \mathrm{KCl}, 10 \mathrm{mM}$ Tris/HCl (pH 9.0), $0.01 \%$ Triton-X 100, $1.5 \mathrm{mM} \mathrm{MgCl}{ }_{2}$ dNTPs ( $50 \mu \mathrm{M}$ each), and $0.1 \mu \mathrm{M}$ of both sense and antisense primers. Amplification was performed as follows: $94^{\circ} \mathrm{C}$ for four minutes, followed by 36 cycles at $94^{\circ} \mathrm{C}$ for one minute, $60^{\circ} \mathrm{C}$ for one minute, and $72^{\circ} \mathrm{C}$ for two minutes. After a final extension period of 10 minutes at 

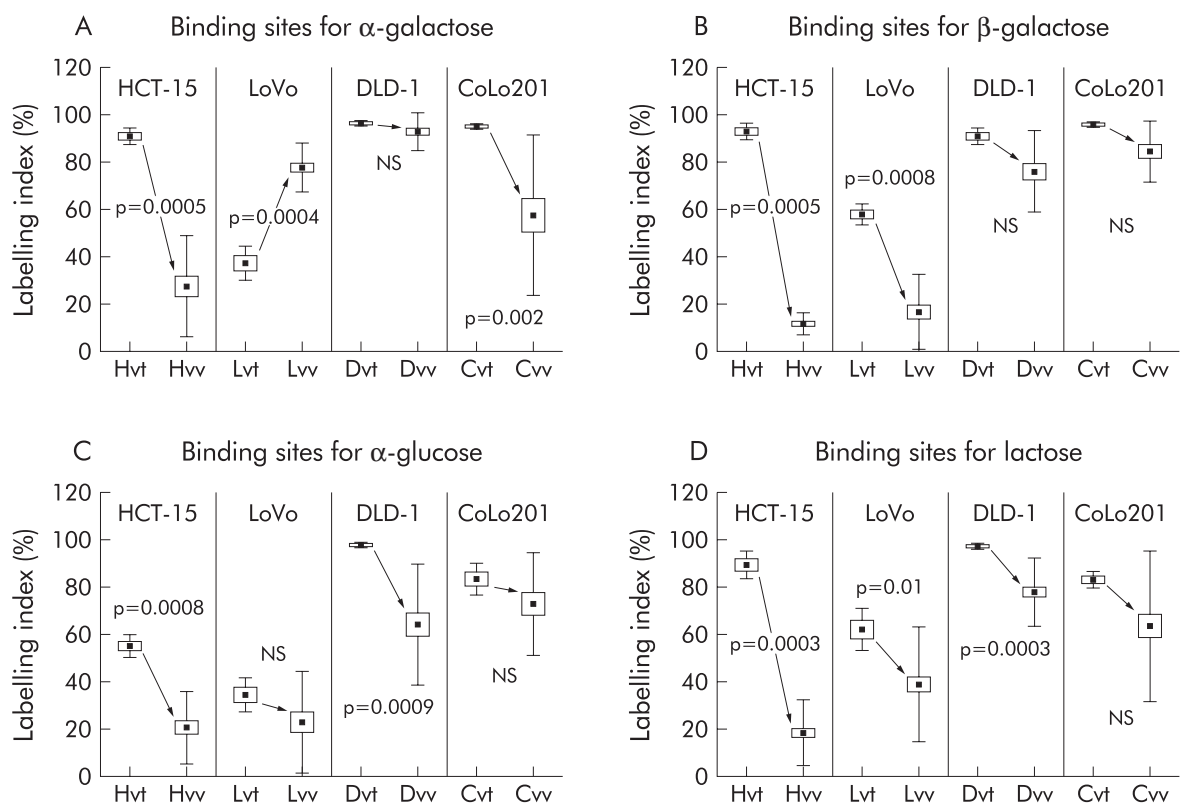

Figure 6 The presence of galactose, glucose, and lactose binding sites in the in vitro versus in vivo human HCT-15, LoVo, DLD-1, and Colo201 colon cancer models was revealed by glycohistochemical probes containing $\alpha$-galactose (A), $\beta$-galactose (B), $\alpha$-glucose (C), and lactose (D) residues covalently attached as $\rho$-aminophenyl derivatives to biotinylated otherwise histochemically inert carrier protein. See legend to fig $2 \mathrm{~B}$ for abbreviations.

$72^{\circ} \mathrm{C}$, the amplified products were separated by gel electrophoresis in $2 \%$ TAE gels and visualised by ethidium bromide staining under UV light.

\section{Quantitative determination of in vitro cell motility}

The influence of galectin- $80.15 \mu \mathrm{g} / \mathrm{ml} / \mathrm{cm}^{2}$ coated onto cell culture supports precoated with Matrigel (Becton Dickinson, Meylan, France), and of $100 \mathrm{ng} / \mathrm{ml}$ antigalectin- 8 antibody added to the culture media for 48 hours (in comparison with control conditions) on migration of living HCT-15, LoVo, CoLo201, and DLD-1 cells was quantitatively determined by a computer assisted phase contrast microscope (Olympus, Antwerp, Belgium) equipped with a CCD camera (Hitachi Denshi, Japan). The technical procedure is detailed elsewhere. ${ }^{26}{ }^{27}$ Briefly, our software enabled each HCT-15, LoVo, CoLo201, or DLD- 1 cell in the colony to be distinguished automatically on the basis of distinct morphological characteristics that in turn enabled them to be identified against the background (in the present case, the plastic Falcon dishes). ${ }^{26}{ }^{27}$ Thus automatic distinction of a cell from its background was followed by a process that enabled the coordinates of its centre of gravity (centroid) to be computed. As this operation was performed every four minutes, the trajectory of each cell centroid could be computed by interpolation. Two motility descriptors were then determined from these trajectories for each of the human colon cancer cells subjected to the computer assisted video microscopy procedure. The first descriptor was related to the average speed of the cells (the quantitative AS variable). ${ }^{26}{ }^{27}$ The second consisted of the maximum relative distance to the origin (the quantitative MRDO variable) of the cells. ${ }^{26}{ }^{27}$ This latter variable was the greatest linear distance found between the original and subsequent positions of each cell, divided by the duration of observation. ${ }^{26}{ }^{27}$ At the beginning of the experiment ( $t=0$ hours), 20-45 malignant colon cells were present in the field of the phase contrast microscope and were thus computed by the CCD camera. At the end of the experiments $(t=48$ hours $)$, trajectories of 130-420 malignant cells had been analysed in each experimental condition; these analyses were carried out in triplicate. The culture media used were identical to those described above.

\section{Statistical analyses}

Statistical comparisons of the data were carried out using the Fisher F test (one way variance analysis for more than two groups) or the Student's $t$ test (for two groups) after checking for variance using the Levene test and for normal distribution of the data using the $\chi^{2}$ test. When these conditions were not satisfied, the non-parametric Kruskall-Wallis (for more than two groups) or the Mann-Whitney (for two groups) tests were carried out. Rank correlation statistical analyses were performed with the Kendall test. All statistical analyses were carried out using the Statistica software package (Statsoft, Tulsa, Oklahoma, USA).

\section{RESULTS}

Galectin-8 expression in human colon tissue samples and in pellets from in vitro cultured human colon cancer cell lines and their corresponding in vivo nude mice xenografts

Figure 1 shows that immunohistochemical expression of galectin- 8 appeared to be higher in normal cases (fig 1A) and adenomas (fig 1B) than in carcinomas, regardless of whether tumours were locally (TINOM0; fig IC) or extensively (T4N2M+; fig 1D) invasive. Figure 1 also shows that while galectin-8 was located in both the cytoplasm and nuclei of normal and benign colon tissue, it was located exclusively in the cytoplasm of malignant colon cells.

Each of the 41 human colon tissue samples was subjected to a computer assisted microscope analysis that enabled the percentage of galectin-8 immunopositive cells (LI variable) and galectin-8 concentration per monitored cell (MOD) to be quantitatively determined. Ten distinct fields were analysed for each of the 41 samples, with only epithelial cells being taken into account. Connective tissue was positive for galectin-8 but no statistically significant differences were observed across the various histopathological groups (data not shown). Almost all cases (except No 3) exhibited 90-100\% galectin-8 immunopositive epithelial cells, regardless of histopathological group (data not shown). In contrast, galectin-8 concentration was lower in malignant than in nonmalignant (including normal and dysplastic) colon cases 


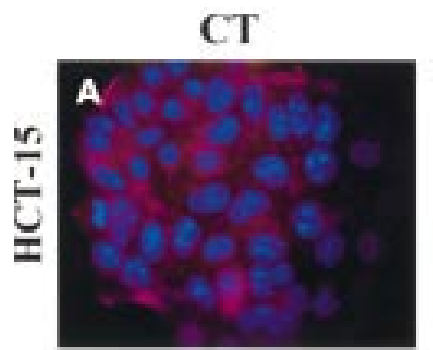

Gal-8 $10 \mathrm{ng} / \mathrm{ml}$
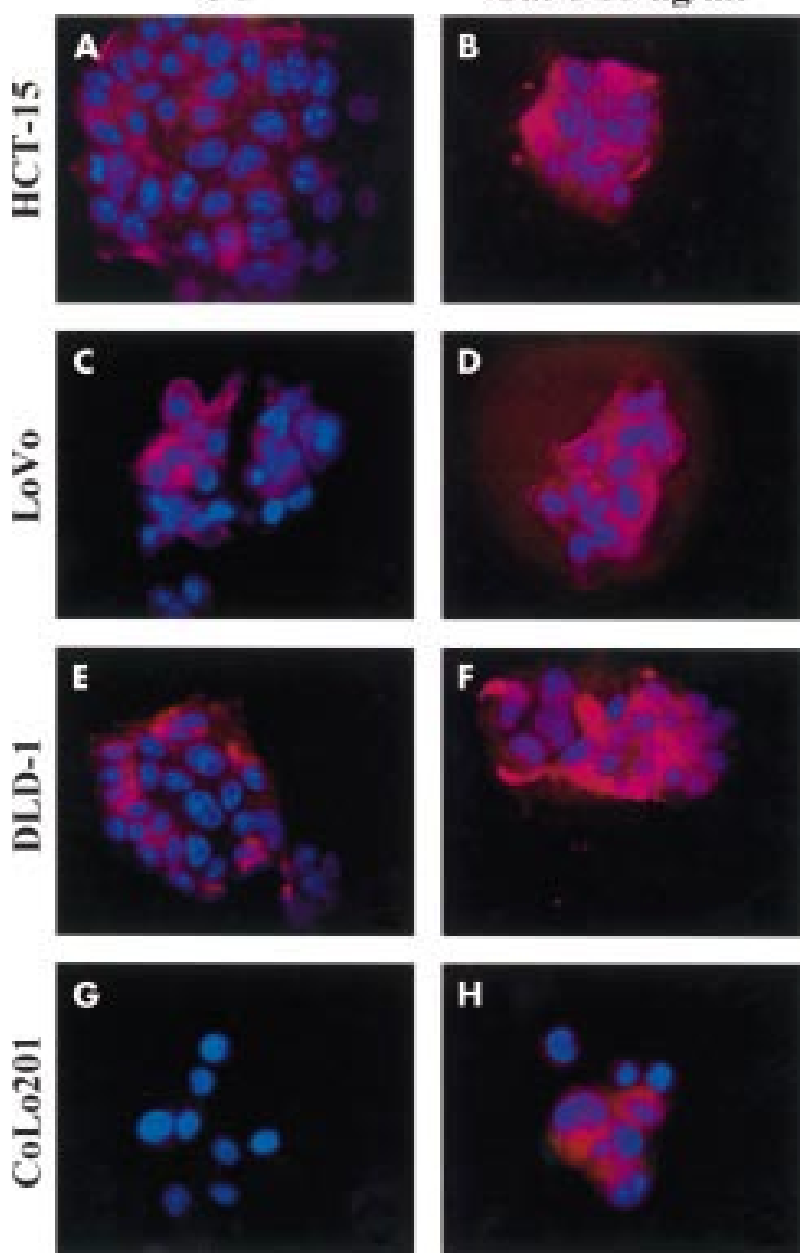

Figure 7 Illustration of galectin-8 binding pattern on human colon cancer cells. Galectin-8 was revealed by fluorescence microscopy with the antigalectin-8 antibody used throughout the study. The four figures on the left (CT panel) illustrate galectin-8 binding patterns in the four cell lines in the culture media to which no exogenous galectin-8 was added. In contrast, the four figures on the right (Gal-8 $10 \mathrm{ng} / \mathrm{ml}$ panel) correspond to experimental conditions in which 10 $\mathrm{ng} / \mathrm{ml}$ galectin- 8 was added 30 minutes before its visualisation by means of the fluorescent antigalectin- 8 antibody.

(data not shown). Consequently, for the statistical analyses, we grouped together all cases in the same histopathological group and considered only MOD (fig 2A) as LI was not discriminatory.

Figure $2 \mathrm{~A}$ summarises the data and shows that galectin- 8 expression increased from the normal to dysplastic stage, and then decreased markedly from these non-malignant cases to the locally ( $\mathrm{Tl}$ and T2/N0 and M0 cases) and extensively (T3 and $\mathrm{T} 4 / \mathrm{N}+$ and $\mathrm{M}+$ ) invasive carcinomas.

As cell lines offer the chance of extending our analysis to biological cell assays, we investigated four human colon cancer models. Figure 3 illustrates the data obtained using a specific primer set for RT-PCR analysis. In addition to the expected product of $608 \mathrm{bp}$, additional signals for insertional isoforms were invariably present (fig 3 ). Detailed restriction analysis and sequencing for another tandem repeat type galectin had already revealed that an extended transcript codes for a galectin-9 isoform with a 32 amino acid extension of the link peptide. ${ }^{38}$

Figure 4 illustrates the morphological patterns of histochemical galectin-8 staining in the four human in vitro HCT-15, CoLo201, LoVo, and DLD-1 colon cancer cells lines ( fig 4A, C, E, G) and their corresponding in vivo xenografts (fig 4B, D, F, H). These morphological illustrations suggest a decrease

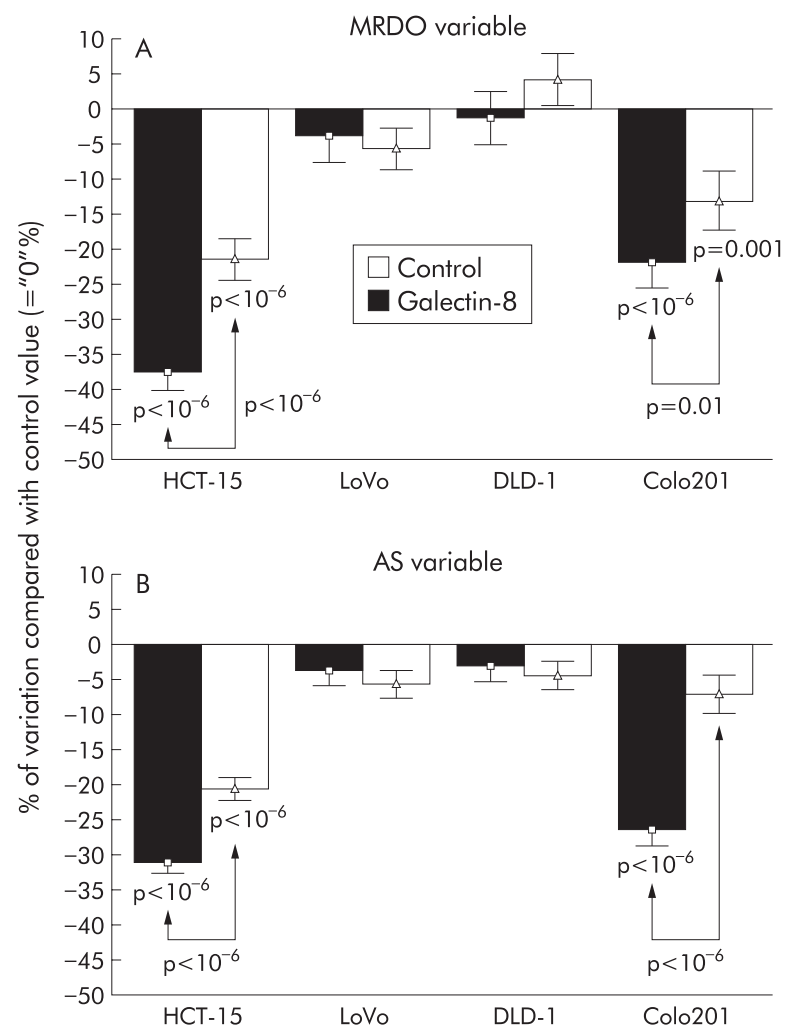

Figure 8 Data obtained by quantitative video microscopy to compute the maximum relative distance from the point of origin (MRDO variable $(A)$ ) and the rate of migration (determined as the average speed (AS) variable (B)) on living HCT-15, LoVo, DLD-1, and CoLo201 human colon cancer cells. The migration levels of these four cell lines were quantitatively assessed in control (arbitrarily defined as " $0 \%$ " and corresponding to culture supports coated with Matrigel) compared with galectin-8 treated conditions in which galectin-8 was coated onto Matrigel coated culture supports. The potential neutralisation of the biological effects induced by galectin- 8 was assayed by adding an antigalectin- 8 antibody to the culture media at a concentration of $100 \mathrm{ng} / \mathrm{ml}$. The experiments lasted 48 hours. Data are presented as means (SEM).

in immunohistochemical galectin-8 expression in the in vivo models compared with those in vitro from which they were derived.

Figure $2 \mathrm{~B}$ illustrates the data obtained from the in vitro cell lines (the " $\mathrm{x}$ "_vt symbols on the $\mathrm{x}$ axis) versus the corresponding in vivo cell lines (the " $\mathrm{x}$ "_ $\mathrm{vv}$ symbols on the $\mathrm{x}$ axis). While significant variations were observed for galectin-8 in epithelial tumours in all models, marked decreases in the extent of galectin-8 expression occurred systematically when the in vivo models were compared with those in vitro from which they were derived (fig 2B).

\section{Immunohistochemical expression of the binding sites for $\alpha$-galactose, $\beta$-galactose, $\alpha$-glucose, and lactose residues in in vitro human colon cancer cell lines and their corresponding in vivo xenografts}

As we observed that the level of galectin-8 expression decreased markedly in in vivo compared with in vitro human colon cancer models, we investigated whether this feature was specific to galectin- 8 or whether it occurred with other $\alpha$ - and $\beta$-galactoside, $\alpha$-glucose, or lactose binding proteins. To reveal the presence of any type of galactoside, glucoside, or lactoside binding protein in these in vitro as opposed to in vivo human colon cancer models, we used glycohistochemical probes containing an $\alpha$-galactose, $\beta$-galactose, $\alpha$-glucose, or a lactose derivative covalently attached to a biotinylated and histochemically inert carrier (that is, bovine serum albumin) which 
enabled the binding capacity to be assessed in situ for these ligands. Galectins can bind $\alpha$ - and $\beta$-galactose at the level of the anomers of the monosaccharide and $\beta$-linked disaccharides with galactose at the non-reducing end. Figure 5 illustrates the histochemical binding patterns of four neoglycoconjugates in the human HCT-15 colon cancer cell line. Figure $5 \mathrm{~A}, 5 \mathrm{C}, 5 \mathrm{E}$, and $5 \mathrm{G}$ relate to the in vitro pellets, while fig $5 \mathrm{~B}, 5 \mathrm{D}, 5 \mathrm{~F}$, and $5 \mathrm{H}$ refer to the nude mice xenografts. Figures $5 \mathrm{~A}$ and $5 \mathrm{~B}$ relate to the $\alpha$-Gal-neoglycoconjugate, and fig 5C and $5 \mathrm{D}$ to the $\beta$-Gal-neoglycoconjugate. Figure $5 \mathrm{E}$ and $5 \mathrm{~F}$ consider the $\beta$-Glu-neoglycoconjugate, and fig $5 \mathrm{G}$ and $5 \mathrm{H}$ the Lac-neoglycoconjugate. The data illustrated in fig 6 clearly indicate that the number of $\alpha$-galactoside (fig 6A), $\beta$-galactoside (fig 6B), $\alpha$-glucoside (fig 6C), and lactoside (fig $6 \mathrm{D})$ binding sites decreased dramatically in the in vivo human colon cancer xenograft compared with levels observed in the corresponding in vitro models. Thus the in vitro to in vivo passage involving a marked decrease in galectin- 8 expression was not restricted to galectin-8 (fig 2B) but affected binding abilities of these other ligands (fig 6).

\section{Extracellular binding of galectin-8 on human colon cancer cells}

Figure 7 illustrates the patterns of binding and staining intensity for galectin-8 in the four colon cancer cell lines. The presence of galectin- 8 was revealed (under fluorescence microscopy) by the antigalectin- 8 antibody used throughout our study. The experimental conditions are detailed in the legend to fig 8 . The data reported here clearly indicate that galectin- 8 binds significantly to human colon cancer cells. These observations validate the fact that we added exogenously galectin-8 (by coating it onto the culture supports) in our experiments on cell motility (see fig 8).

\section{Galectin-8 induced modifications to the in vitro migration levels of the human HCT-15, LoVo, DLD-1, and CoLo201 colon cancer cell lines}

When coated onto a Matrigel culture support, galectin-8 markedly decreased migration levels (in comparison with culture supports also coated with Matrigel but devoid of galectin-8) in the HCT-15 and CoLo201 human colon cancer cell lines but not in the LoVo and DLD-1 cell lines (fig 8). The galectin-8 induced influence on HCT-15 and CoLo201 cells was apparent in both the greatest distance covered by the cells (MRDO variable, fig 8A) and migration rate (AS variable, fig $8 \mathrm{~B}$ ). Addition of the antigalectin- 8 antibody used to immunohistochemically detect galectin- 8 in the in vitro (fig 4A, C, E, G, and fig 8 ) and in vivo (fig 4B, D, F, H) colon cancer models partially neutralised but did not abolish the biological effect induced by galectin-8 (fig 8 ). These results strongly suggest that the galectin- 8 induced decrease in HCT-15 and CoLo201 cell migration actually relates to galectin-8. The fact that the antigalectin- 8 antibody did not totally eliminate galectin- 8 induced effects may be due, at least in part, to the fact that the dose of antigalectin- 8 antibody used was too low. The reason for this may relate to effect of the antibody on cell kinetics. While at the $100 \mathrm{ng} / \mathrm{ml}$ concentration the antigalectin-8 antibody did not significantly modify HCT-15 or CoLo201 cell growth kinetic (data not shown), at higher doses it did (data not shown). In these experiments, we wished to deliberately limit ourselves to antigalectin- 8 antibody concentrations acting on cell migration features only. Non-immune serum was used as a control for the cell motility experiments with the antigalectin- 8 antibody. No significant modifications to colon cancer cell motility were observed with this non-immune serum (data not shown).

Relationship between immunohistochemical galectin-8 expression in human colon cancer xenografts and their growth rate

Galectin-8 caused a marked reduction in migration levels in the HCT-15 and CoLo201 models while having no apparent
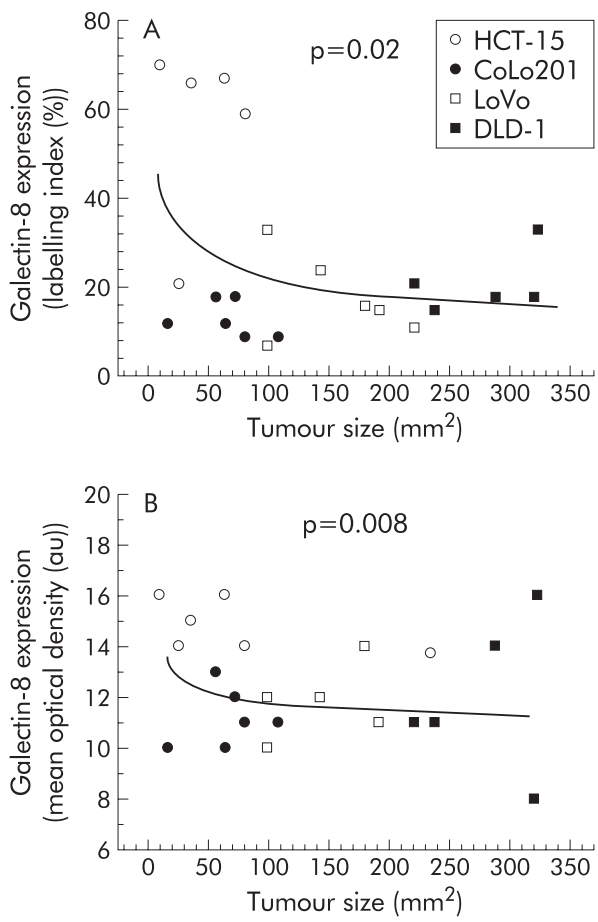

Figure 9 Percentage of immunopositive galectin-8 epithelial colon cancer cells (A) and immunohistochemically detected galectin-8 concentration in epithelial colon cancer cells (B) in relation to growth rate in the HCT-15, CoLo201, LoVo, and DLD-1 human colon cancer cell lines grafted into nude mice.

effect on LoVo or DLD-1, even though the four models uniformly produced galectin-8 mRNA (fig 4) and galectin-8 protein (figs 4,7 ). In addition to cell motility, growth rate was examined for correlations with galectin-8 expression. Indeed, levels of galectin-8 expression were related significantly and inversely to the growth rate of these four models when xenografted in vivo into nude mice: both the percentage of immunopositive epithelial cells (fig 9A) and galectin-8 concentration in these epithelial tumour cells (fig 9B) were significantly higher in the slowly growing HCT-15 and CoLo201 colon cancer models than in the rapidly growing LoVo and DLD-1 cell lines. Thus galectin- 8 significantly reduced the migration rates in the HCT-15 and CoLo201 models only and not in the LoVo and DLD-1 models (fig 7), indicating a sensitivity related to the growth rate of these colon cancer models (fig 9).

\section{DISCUSSION}

In this study we found that a marked decrease in immunohistochemical galectin-8 expression occurred with the development of malignancy in human colon tissue. Malignant colon tissue exhibits a significantly lower galectin-8 concentration than normal or benign tissue within colon cancers, and those with an extensive invasion capacity $(\mathrm{T} 3-4 / \mathrm{N}+/ \mathrm{M}+)$ harbour significantly less galectin- 8 than colon cancers with a localised invasion capacity (T1-2/N0/M0). The four experimental models (HCT-15, LoVo, CoLo201, and DLD-1) showed more intense galectin-8 dependent staining in vitro than in vivo. Grafting the four experimental human colon cancer models into nude mice enabled us to show that the immunohistochemical expression of galectin- 8 was inversely related to the growth rate of the tumours. In vitro, galectin- 8 reduced the migration rate of only those human experimental models (HCT-15 and CoLo201) which exhibited the lowest growth rate in vivo.

To date, analysis of galectins in tumour biology has almost exclusively focused on galectin-1 and galectin-3. To fully 
understand the protein (lectin)-carbohydrate interaction, it is essential to analyse the presence and functional aspects of the other family members. At present, only three reports relate to galectin-8, with initial cloning work of rat and human proteins, ${ }^{192139}$ and binding to the $\alpha 3 \beta 1$ integrin has been shown. ${ }^{40}$ As detailed in the introduction, galectin- 8 resembles galectin-4 and galectin- 6 in many respects but it also has several unique features. Interestingly, our PCR data for the four human colon cancer cell lines revealed the presence of additional bands in the gels (see fig 3), corroborating earlier observations on the presence of an additional isoform, the related tandem repeat type galectin- $9 .{ }^{38}$

In contrast with the rather insoluble galectin-4, galectin-8 stays in solution and is secreted by a non-classical pathway acting as an extracellular effector. It also binds extracellularly to human colon cancer cells, as illustrated in the present study (see fig 7). This is why we characterised the influence of galectin-8 on migration of human colon cancer cells by coating the cell culture support with galectin-8.

Our data showed that galectin- 8 coated on the culture supports of HCT-15 and CoLo-201 cells remained active and markedly decreased migration levels of these two human colon cancer models for at least 48 hours (see fig 8). Addition of an antigalectin- 8 antibody to the culture media of these HCT-15 and CoLo-201 cell lines significantly neutralised (but did abolish) the galectin-8 induced decrease in HCT-15 and CoLo-201 cell migration. In contrast, neither galectin-8 nor antigalectin-8 antibody significantly modified migration levels of the two other human colon cancer models (LoVo and DLD-1) at the concentrations tested (see fig 8).

The subcutaneous grafting procedure of the four human colon cancer cell lines onto nude mice revealed that LoVo and DLD-1, the two rapidly growing models, exhibited significantly lower amounts of galectin-8 than HCT-15 and CoLo201 (see fig 9), the two slowly growing models. Thus comparing these in vivo data with the abovementioned in vitro data it appears that on the basis of an as yet unknown mechanism, galectin- 8 exerts an inhibitory influence on the migration of slowly growing human colon cancer cells only, and not on that of rapidly growing ones. One reason for the differential sensitivity could relate to the action of proteases on ligand sites which may include the extracellular matrix proteins laminin or fibronectin, and certain integrins.

These observations concerning both the in vitro and in vivo experimental models are corroborated by clinical data. Indeed, quantitative determination of the immunohistochemical expression of galectin- 8 in the series of clinical specimens clearly shows that the extensively invasive colon cancers (associated with a high TNM level) exhibited significantly less galectin-8 than locally invasive ones (associated with a low TNM level) (see fig 2). Colon cancers associated with a high TNM level are thought to express significantly higher MMP levels than colon cancers associated with a low TNM level. ${ }^{41-44}$ The in vivo decrease in galectin-8 expression compared with the in vitro situation has a precedent in the case of murine P388D ${ }_{1}$ cells and galectin-1..$^{45}$ Similarly, the exclusively cytoplasmic localisation with no further nuclear presence has been observed in later phases of colon tumour progression for galectin-3. ${ }^{46}$ To infer an overall capacity to bind sugar ligands, we used glycohistochemical probes and noted a decrease, arguing in favour of loss of binding capacity. This modulation should be considered in functional analyses of the in vitro and in vivo data for the same cell type. Galectin- 8 will most likely home in on $\beta$-galactosides as a first step to elicit biological cell activities. Interestingly, neither galectin-1 nor galectin-3 (for review see André and colleages ${ }^{47}$ ) inhibited 1299 cell adhesion, indicating differential selection of target molecules and/or affinity among galectins. For galectin-8, blotting revealed $\alpha 3$, $\alpha 6$, and $\beta 1$ integrins as key cell components. ${ }^{40}$ Moreover, its design, with two carbohydrate recognition domains in one molecule, is evocative of that of homodimeric galectin-l whose binding to ganglioside GMl and cross linking have been involved in the negative growth regulation of cultured human neuroblastoma cells. ${ }^{48} \mathrm{~A}$ further comment pertains to our previous observations that plant lectins binding $\alpha$ - or $\beta$-galactose residues significantly modify the growth level in HCT- 15 and LoVo in vitro models. ${ }^{32}$ However, as recently shown histochemically for VAA, RCA, and galectin-1 in large intestine tissue, ${ }^{49}$ the precise specificity of plant and mammalian lectins may differ despite the same monosaccharide target. These observations and our present study indicate that an endogenous lectin can serve as instructive guidelines for detecting functional protein (lectin)-glycan recognition systems.

In conclusion, the present study illustrates the importance of extending our research beyond galectin- 1 and -3 in tumour biology. We showed that the level of galectin-8 expression decreased markedly during tumorigenesis in human colon tissue. While assessing cell migration, galectin- 8 significantly reduced these processes in slowly growing human colon cancers. In summary, the present study highlights the fact that the diagnostic and/or prognostic value of galectins in colon tissue cannot be restricted to galectin- 1 and galectin-3.

\section{ACKNOWLEDGEMENTS}

IC is the holder of a grant from the "Fondation Yvonne Boël" and the "Fondation Rose et Jean Hoguet"; RK is a senior research associate with the Fonds National de la Recherche Scientifique (FNRS, Belgium). The present study was carried out with the support of grants awarded by the "Fonds de la Recherche Scientifique Médicale" (FRSM, Belgium), "Les Amis de l'Insitut Bordet" (Brussels, Belgium), and the Dr M Scheel-Stiftung für Krebsforschung (Bonn, Germany).

\section{Authors' affiliations}

N Nagy, I Salmon, Laboratory of AnatomoPathology, Erasmus

University Hospital, Free University of Brussels (ULB), Brussels, Belgium

Y Bronckart, I Camby, A Danguy, R Kiss, Laboratory of

Histopathology, Faculty of Medicine, Free University of Brussels (ULB), Brussels, Belgium

H Legendre, J-C Pector, Department of Surgery, Jules Bordet Institute, Free University of Brussels (ULB), Brussels, Belgium

H Lahm, Institute of Molecular Animal Breeding, Gene Centre

Ludwig-Maximilians-University, Munich, Germany

H Kaltner, H-J Gabius, Institute of Physiological Chemistry, Faculty of Veterinary Medicine, Ludwig-Maximilians-University, Munich, Germany Y Hadari, Department of Chemical Immunology, Weizmann Institute of Science, Rehovot, Israel

P Van Ham, Laboratory of Logical and Numerical Systems, Faculty of Applied Sciences, Free University of Brussels (ULB), Brussels, Belgium

P Yeaton, Digestive Health Centre, University of Virginia Health System, Charlottesville, Virginia, USA

Y Zick, Department of Molecular Cell Biology, Weizmann Institute of Science, Rehovot, Israel

\section{REFERENCES}

1 Gabius HJ. Endogenous lectins in tumors and the immune system. Cancer Invest 1987;5:39-46.

2 Raz A, Lotan R. Endogenous galactoside-binding lectins: a new class of functional tumor cell surface molecules related to metastasis. Cancer Metastasis Rev 1987;6:433-52.

$3 \mathrm{Kim}$ YJ, Varki A. Perspective on the significance of altered glycosylation of glycoproteins in cancer. Glycoconjugate J 1997;14:569-76.

4 Hakomori SI. Cancer-associated glycosphingolipid antigens: their structure, organisation, and function. Acta Anat 1998;161:79-90.

5 Reuter G, Gabius HJ. Eukaryotic glycosylation: whim of nature or multipurpose tool? Cell Mol Life Sci 1999;55:368-22.

6 Barondes SH, Cooper DN, Gitt MA, et al. Galectins. Structure and function of a large family of animal lectins. J Biol Chem 1994;269:20807-10

7 Hirabayashi J, ed. Recent topics on galectins. Trends Glycosci Glycotechnol 1997;9:1-180

8 Itzkowitz SH. Galectins: Multipurpose carbohydrate-binding proteins implicated in tumor biology. Gastroenterology 1997:113:2003-5. 9 Gabius HJ. Animal lectins. Eur J Biochem 1997;243:543-76.

10 Kaltner H, Stierstorfer B. Animal lectins as cell adhesion molecules. Acta Anat 1998; 161:162-79.

11 Perillo NL, Marcus ME, Baum LG. Galectins: versatile modulators of cell adhesion, cell proliferation, and cell death. J Mol Med 1998;76:402-12. 
12 Hughes RC. Secretion of the galectin family of mammalian carbohydrate-binding proteins. Biochim Biophys Acta 1999;1473:17285.

13 Rabinovich GA. Galectins: an evolutionarily conserved family of animal lectins with multifunctional properties; a trip from the gene to clinical therapy. Cell Death Differ 1999;6:71 1-21.

14 Gabius HJ. Concepts of tumor lectinology. Cancer Invest 1997; 15:454-64.

15 Bresalier RS, Mazurek N, Sternberg LR, et al. Metastasis of human colon cancer is altered by modifying expression of the $\beta$-galactoside-binding protein galectin-3. Gastroenterology 1998;115:287-96.

16 Nakamura $M$, Inufusa $H$, Adachi T, et al. Involvement of galectin-3 expression in colorectal cancer progression and metastasis. Int J Oncol 1999;15:143-8.

17 Schwarz G, Remmelink M, Decaestecker C, et al. Galectin fingerprinting in tumor diagnosis. Differential expression of galectin-3 and galectin-3-binding sites, but not galectin-1, in benign versus malignant uterine smooth muscle tumors. Am J Clin Pathol 1999;111:623-31.

18 Lahm H, André S, Höflich A, et al. Comprehensive galectin fingerprinting in a panel of 61 human tumor cell lines by RT-PCR and its implications for diagnostic and therapeutic procedures. J Cancer Res Clin Oncol 2001;127:375-386.

19 Hadari YR, Paz K, Dekel R, et al. Galectin-8. A new rat lectin, related to galectin-4. J Biol Chem 1995;270:3447-53.

20 Rechreche H, Mallo GV, Montalto G, et al. Cloning and expression of the mRNA of human galectin-4, an S-type lectin down-regulated in colorectal cancer. Eur J Biochem 1997;248:225-30.

21 Hadari YR, Eisenstein M, Zakut R, et al. Galectin-8: on the road from structure to function. Trends Glycosci Glycotechnol 1997;9:103-12.

22 Gitt MA, Colnot C, Poirier F, et al. Galectin-4 and galectin-6 are two closely related lectins expressed in mouse gastrointestinal tract. J Biol Chem 1998;273:2954-60

23 Oda Y, Herrmann J, Gitt MA, et al. Soluble lactose-binding lectin from rat intestine with two different carbohydrate-binding domains in the same peptide chain. J Biol Chem 1993:268:5929-39.

24 Huflejt ME, Jordan ET, Gitt MA, et al. Strikingly different localization of galectin-3 and galectin-4 in human colon adenocarcinoma T84 cells. Galectin-4 is localized at sites of cell adhesion. J Biol Chem 1997;272: 14294-303.

25 Danielsen EM, Van Deurs B. Galectin-4 and small intestinal brush border enzymes form clusters. Mol Biol Cell 1997;8:2241-51.

26 DeHauwer C, Camby I, Darro F, et al. Dynamic characterization of glioblastoma cell motility. Biochem Biophys Res Commun 1997; 232:267-72.

27 DeHauwer C, Camby I, Darro F, et al. Gastrin inhibits motility, decreases cell death and increases cell proliferation in human glioblastoma cell lines. J Neurobiol 1998;37:373-82.

28 Bronckart Y, Nagy N, Decaestecker C, et al. Grading dysplasia in colorectal adenomas by means of the quantitative binding pattern determination of Arachis hypogaea, Dolichos biflorus, Amaranthus caudatus, Maackia amurensis and Sambucus nigra agglutinins. Hum Pathol 1999;30:1 178-91.

29 Beahrs OH, Henson DE, Hutter RVP, et al eds. American Joint Committee on Cancer. Manual for Staging of Cancer, 4th edn. Philadelphia: JB Lippincott, 1992:1-82.

30 Darro F, Kruczynski A, Etiévant C, et al. Characterization of the differentiation of human colorectal cancer cell lines by means of Voronoi diagrams. Cytometry 1993;14:783-92.

31 Darro F, Camby I, Kruczynski A, et al. Characterization of the influence of anti-gastrin, anti-epidermal growth factor, anti-oestradiol, and anti-luteinizing hormone releasing hormone antibodies on the proliferation of 27 cell lines from the gastrointestinal tract. Gut 1995;36:220-30.
32 Kiss R, Camby I, Duckworth C, et al. In vitro influence of Phaseolus vulgaris, Griffonia simplicifolia, concanavalin A, wheat germ, and peanut agglutinins on HCT-15, LoVo, and SW837 human colorectal cancer cell growth. Gut 1997;40:253-61.

33 Jannot MC, Kruczynski A, Limouzy A, et al. Spontaneous evolution of cytoplasmic lectin binding and nuclear size and deoxyribonucleic acid content in human colorectal cancers grafted onto nude mice. Lab Invest 1993:68:446-55

34 Danguy A, Kayser K, Bovin NV, et al. The relevance of neoglycoconjugates for histology and pathology. Trends Glycosci Glycotechnol 1995;7:261-75.

35 Gabius HJ, Bardosi A. Neoglycoproteins as tools in glycohistochemistry. Progr Histochem Cytochem 1991;22:1-66.

36 Camby I, Nagy N, Lopes MB, et al. Supratentorial pilocytic astrocytomas, astrocyłomas, anaplastic astrocyłomas and glioblastomas are characterized by a differential expression of $\mathrm{S} 100$ proteins. Brain Pathol 1999;9:825-43.

37 Lahm H, Amstad P, Yilmaz Z, et al. Interleukin-4 down-regulates expression of c-kit and autocrine stem cell factor in human colorectal carcinoma cells. Cell Growth Differ 1995;6:1111-18.

38 Lahm H, Höflich A, André S, et al. Gene expression of galectin-9/ecalectin, a potent eosinophil chemoattractant, and/or the insertional isoform in human colorectal carcinoma cell lines and detection of frame-shift mutations for protein sequence truncations in the second functional lectin domain. Int J Oncol 2000;17:519-24.

39 Su ZZ, Lin J, Shen R, et al. Surface-epitope masking and expression cloning identifies the human prostate carcinoma tumor antigen gene PCTA-1 a member of the galectin gene family. Proc Natl Acad Sci USA 1996;93:7252-7.

40 Hadari YR, Rinat AG, Levy $Y$, et al. Galectin-8 binding to integrins inhibits cell adhesion and induces apoptosis. J Cell Sci 2000;113: 2385-97.

41 Murray GI, Duncan ME, O'Neil P, et al. Matrix metalloproteinase-1 is associated with poor prognosis in colorectal cancer. Nat Med 1996;2:461-2.

42 Zeng ZS, Cohen AM, Guillem JG. Loss of basement membrane type IV collagen is associated with increased expression of metalloproteinases 2 and 9 (MMP-2 and MMP-9) during human colorectal tumorigenesis. Carcinogenesis 1999;20:749-55.

43 Garbett EA, Reed MW, Brown NJ. Proteolysis in human breast and colorectal cancer. Br J Cancer 1999;81:287-93.

44 Adachi Y, Yamamoto $\mathrm{H}$, Itoh $\mathrm{F}$, et al. Contribution of matrilysin (MMP-7) to the metastatic pathway of human colorectal cancers. Gut 1999;45:252-8.

45 Gabius HJ, Vehmeyer K. Effect of microenvironment and cell-line type on carbohydrate-binding proteins of macrophage-like cells. Biochem Cell Biol 1988;66:1169-76.

46 Sanjuan X, Fernandez PL, Castells A, et al. Differential expression of galectin-3 and galectin-1 in colorectal cancer progression. Gastroenterology 1997;113:1906-18.

47 André S, Kojima S, Yamazaki N, et al. Galectins-1 and -3 and their ligands in tumor biology. J Cancer Res Clin Oncol 1999;125:461-74.

48 Kopitz J, von Reitzenstein C, Burchert M, et al. Galectin-1 is a major receptor for ganglioside $\mathrm{GM}_{1}$, a product of the growth-controlling activity of a cell surface ganglioside sialidase, on human neuroblastoma cells in culture. J Biol Chem 1998;273:11205-11.

49 Brinck U, Korabiowska M, Bosbach $R$, et al. Detection of inflammationand neoplasia-associated alterations in human large intestine using plant/invertebrate lectins, galectin-1 and neoglycoproteins. Acta Anat $1998 ; 161: 219-33$ 